\title{
Treatment with the PPAR $\gamma$ Agonist Pioglitazone in the Early Post-ischemia Phase Inhibits Pro-inflammatory Responses and Promotes Neurogenesis Via the Activation of Innate- and Bone Marrow-Derived Stem Cells in Rats
}

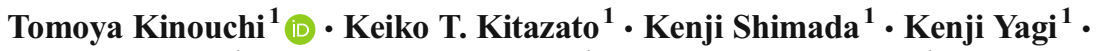 \\ Yoshiteru Tada $^{1} \cdot$ Nobuhisa Matsushita $^{1} \cdot$ Yoshitaka Kurashiki $^{1} \cdot$ Junichiro Satomi $^{1}$ • \\ Masataka Sata $^{2} \cdot$ Shinji Nagahiro ${ }^{1}$
}

Received: 20 December 2016/Revised: 30 September 2017 / Accepted: 5 October 2017 / Published online: 6 November 2017

(C) The Author(s) 2017, corrected publication November/2017. This article is an open access publication

\begin{abstract}
Neurogenesis is essential for a good post-stroke outcome. Exogenous stem cells are currently being tested to promote neurogenesis after stroke. Elsewhere, we demonstrated that treatment with the PPAR $\gamma$ agonist pioglitazone (PGZ) before cerebral ischemia induction reduced brain damage and activated survival-related genes in ovariectomized (OVX) rats. Here, we tested our hypothesis that post-ischemia treatment with PGZ inhibits brain damage and contributes to neurogenesis via activated stem cells. Bone marrow (BM) cells of 7-week-old Wistar female rats were replaced with $\mathrm{BM}$ cells from green fluorescent protein-transgenic $\left(\mathrm{GFP}^{+}\right.$ ${ }^{\mathrm{BM}}$ ) rats. Three weeks later, they were ovariectomized $\left(\mathrm{OVX} / \mathrm{GFP}^{+\mathrm{BM}}\right.$ rats). We subjected 7-week-old Wistar male and 13-week-old $\mathrm{OVX} / \mathrm{GFP}^{+\mathrm{BM}}$ rats to 90 -min cerebral ischemia. Male and $\mathrm{OVX} / \mathrm{GFP}^{+\mathrm{BM}}$ rats were divided into two groups, one was treated with PGZ $(2.5 \mathrm{mg} / \mathrm{kg} /$ day $)$ and the other served as the vehicle control (VC). In both male and $\mathrm{OVX} / \mathrm{GFP}^{+\mathrm{BM}}$ rats, post-ischemia treatment with PGZ reduced neurological deficits and the infarct volume. In male rats, PGZ decreased the mRNA level of IL-6 and M1-like macrophages after $24 \mathrm{~h}$. In OVX/GFP ${ }^{+\mathrm{BM}}$ rats, PGZ augmented the proliferation of resident stem cells in the subventricular zone (SVZ) and the recruitment of $\mathrm{GFP}^{+\mathrm{BM}}$ stem cells on days
\end{abstract}

Tomoya Kinouchi

k-tomoya@xg8.so-net.ne.jp

1 Department of Neurosurgery, Institute of Biomedical Sciences, Tokushima University Graduate School, 3-18-15, Kuramoto-cho, Tokushima 770-8503, Japan

2 Department of Cardiovascular Medicine, Institute of Biomedical Sciences, Tokushima University Graduate School, Tokushima, Japan
7-14. Both types of proliferated stem cells migrated from the SVZ into the peri-infarct area. There, they differentiated into mature neurons, glia, and blood vessels in association with activated Akt, MAP2, and VEGF. Post-ischemia treatment with PGZ may offer a new avenue for stroke treatment through contribution to neuroprotection and neurogenesis.

Keywords Neurogenesis · Post-stroke treatment · PPAR $\gamma$. Resident stem cells $\cdot$ Bone marrow-derived stem cells

\section{Introduction}

Brain ischemia is a leading cause of morbidity and mortality [1]. Although thrombolysis for stroke is an accepted treatment, fewer than $5 \%$ of stroke patients are treated with tissue plasminogen activator. New therapeutic strategies are needed for stroke patients.

While cerebral ischemia induces pro-inflammatory cytokines, resulting in the expansion of brain damage, it stimulates inherent neurogenesis in the subventricular zone (SVZ) and promotes the migration of newly formed neuronal progenitor cells toward the ischemic area [2]. This process may help to repair the brain and reconstruct neural networks. In rodents [3], monkeys [4], and humans [5], the SVZ, located in the lateral wall lining the lateral ventricle, harbors the largest population of neural stem cells capable of generating new neurons, astrocytes, and oligodendrocytes. While enhanced neurogenesis and angiogenesis in the SVZ and subgranular zone have been documented in adult rodent brains after focal ischemia [6], as most of the newly formed cells promptly undergo apoptosis, their effect on functional recovery is limited [2]. 
The administration of pioglitazone (PGZ), a peroxisome proliferator-activated receptor gamma (PPAR $\gamma$ ) agonist, before cerebral ischemia induction protected rodents against ischemic brain damage [7, 8]. Its beneficial effects were associated with a decrease in the expression of interleukin-6 (IL-6) and caspase-3 and the improvement of neurological function in male rats [7]. However, there are few studies regarding the effects of post-ischemic treatment with PGZ.

As estrogen deficiency and menopause are thought to be major risk factors for cerebral ischemia, in an earlier study [8], we compared the effects of pre-treatment with PGZ on cerebral ischemia in oophorectomized (OVX) and non-OVX rats. We demonstrated that PGZ prevented ischemic brain damage in OVX rats when it was administered before stroke and that this was associated with the upregulation of anti-apoptotic and survival genes via the trans-activation of STAT3 and PPAR $\gamma$ in the peri-infarct region. This suggests its contribution to neurogenesis. However, it remained to be determined whether postischemic treatment with PGZ contributes to neurogenesis and neurological improvement after an ischemic insult.

The local release of PGZ promoted wound healing [9]; the regulation of monocytes/macrophages by PGZ inhibited plaque destabilization and rupture in $\mathrm{ApoE}^{-/-}$mice [10], suggesting that post-stroke treatment with PGZ may be useful. Based on findings in other [7, 9, 10] and our studies [8], we hypothesized that the activation of PPAR $\gamma$ elicited by the administration of PGZ after stroke may be beneficial due to not only its anti-inflammatory effects but also its promotion of neurogenesis. As neurogenesis is regulated by the stem cell niche [11], we focused on this issue. To examine whether PGZ is associated with neuro- and angiogenesis, we prepared female rats whose bone marrow (BM) cells were replaced with $\mathrm{BM}$ cells from green fluorescent protein-transgenic $\left(\mathrm{GFP}^{+\mathrm{BM}}\right)$ rats before oophorectomy $\left(\mathrm{OVX} / \mathrm{GFP}^{+\mathrm{BM}}\right.$ rats). We first examined the neuroprotective effects elicited by post-ischemia treatment with $\mathrm{PGZ}$ in male and $\mathrm{OVX} / \mathrm{GFP}^{+}$ ${ }^{\mathrm{BM}}$ rats and then investigated its effects on neurogenesis in $\mathrm{OVX} / \mathrm{GFP}^{+\mathrm{BM}}$ rats.

Here, we show that even post-ischemia, early-phase treatment with PGZ inhibits the infarct size and alleviates neurological deficits in male and in $\mathrm{OVX} / \mathrm{GFP}^{+\mathrm{BM}}$ rats. We demonstrate that the decrease in the pro-inflammatory cytokine IL-6 and in M1-like macrophages associated with an increase in PPAR $\gamma$ after ischemia in male rats. We also document that in $\mathrm{OVX} / \mathrm{GFP}^{+\mathrm{BM}}$ rats, $\mathrm{PGZ}$ activated innate stem cells in the SVZ and recruitment of $\mathrm{GFP}^{+\mathrm{BM}}$ stem cells with an increase in PPAR $\gamma$ and then increased the expression of Akt, MAP2, and VEGF in the cortical peri-infarct area, leading to neurogenesis.

\section{Materials and Methods}

Our study was approved by the Ethics Committee of the Institute of Biomedical Sciences, Tokushima University Graduate School, and conducted in accordance with current RIGOR guidelines $[12,13]$ and the National Institutes of Health (NIH) Guide for the Care and Use of Laboratory Animals. We purchased male and female Wistar rats from Charles River Laboratories Japan Inc. (Yokohama, Japan). They were housed in conventional rat cages in a temperature- and humidity-controlled room (about $23{ }^{\circ} \mathrm{C}$ and $50 \%$, respectively) under a 12 -h inverted light/dark cycle and were fed standard chow. Anesthesia was with $2 \%$ isofluorane in $30 \%$ oxygen and $70 \%$ nitrous oxide. The experiments were reported according to the "Animal Research: Reporting of In Vivo Experiments (ARRIVE)" guidelines to improve the design, analysis, and reporting of research using animals - maximizing information published and minimizing unnecessary studies. All procedures were performed by investigators blinded to the treatment that rats had undergone according to a protocol approved by the Animal Care Committee of Tokushima University Medical School. For randomization, we used random-number tables; the treatment groups were identified by ear punch and the cages were labeled.

\section{Whole-Body Irradiation and BM Transplantation in Female Rats}

The BM donors were 6-week-old GFP-transgenic rats purchased from Japan SLC, Inc. (Hamamatsu, Japan). They were killed under deep anesthesia by cervical dislocation. BM was obtained by flushing the femora and tibiae with sterile phosphate-buffered saline (PBS). BM cells were suspended in PBS, washed several times, counted, and resuspended at $4 \times 10^{7}$ cells $/ \mathrm{ml}$. All procedures were as described elsewhere [14].

At the age of 7 weeks, the Wistar female rats underwent whole-body irradiation with a single 10-Gy dose. Within $24 \mathrm{~h}$, thereafter, they were injected with $300 \mu \mathrm{l}$ of the GFP-labeled $\mathrm{BM}$ cell suspension via the tail vein.

\section{Animals}

Females received BM cells from GFP rats who were subjected to bilateral OVX at the age of 10 weeks $\left(\mathrm{OVX} / \mathrm{GFP}^{+\mathrm{BM}}\right.$ rats). Based on our and other earlier studies [7, 8], 7-week-old male and 13-week-old $\mathrm{OVX} / \mathrm{GFP}^{+\mathrm{BM}}$ Wistar rats weighing 250 $270 \mathrm{~g}$ and $280-300 \mathrm{~g}$, respectively, were subjected to 90 min middle cerebral artery occlusion-reperfusion (MCAOR). One group of randomly selected rats was injected intraperitoneally (i.p.) with $2.5 \mathrm{mg} / \mathrm{kg} /$ day PGZ; the other was the 
vehicle control (VC). In male and OVX/GFP ${ }^{+\mathrm{BM}}$ rats, we recorded the effects of PGZ delivered in the early phase after MCAO-R (day 0) and once a day for 7 (males) and 14 consecutive days $\left(\mathrm{OVX} / \mathrm{GFP}^{+\mathrm{BM}}\right.$ rats).

Besides examining the response to early-phase post-ischemia treatment with PGZ in OVX/GFP ${ }^{+\mathrm{BM}}$ rats, we also assessed its effect on post-MCAO-R regeneration on days 7-14. The OVX/GFP ${ }^{+\mathrm{BM}}$ rats were randomly sub-divided into two groups: one group received $2.5 \mathrm{mg} / \mathrm{kg}$ PGZ i.p. once a day for 14 consecutive days after MCAO-R, the other served as the VC. PGZ, a gift from Takeda Pharmaceutical Co., was dissolved in dimethylsulfoxide (DMSO) and diluted $(\times 3)$ with saline just before i.p. injection $(0.4 \mathrm{ml} / \mathrm{kg}$ body weight). $\mathrm{VC}$ rats were injected DMSO dilution at the same concentration and volume as PGZ.

\section{Focal Cerebral Ischemia}

During all surgical procedures, the rats were under anesthesia with $2 \%$ isofluorane in $30 \%$ oxygen and $70 \%$ nitrous oxide; their rectal temperature was monitored with a thermometer (KN-91, Natsume) and maintained at $37 \pm 0.5{ }^{\circ} \mathrm{C}$ with a warming plate.

For 90-min MCAO, we inserted an intraluminal filament as described elsewhere $[15,16]$. To block major collateral flow, the pterygopalatine artery was ligated at its origin. The internal and common carotid artery were transiently occluded with loosely tied 3-0 silk sutures; a silicon-coated 4-0 nylon thread was introduced into the external carotid artery and advanced into the internal carotid artery to occlude the proximal orifice of the MCA. To confirm MCAO, we used a laser-Doppler flow probe (Unique Medical, Osaka, Japan) to measure the blood flow at the temporal bone surface at a site $1 \mathrm{~mm}$ posterior to the bregma and $3 \mathrm{~mm}$ inferior to the temporal line. MCAO reduced the blood flow to $20-30 \%$ of the baseline.

Rats with successful MCAO-R consistently exhibited circling behavior, decreased resistance to lateral push, forelimb flexion, and shoulder adduction. We excluded around $10 \%$ of the rats because MCAO-R was incomplete. Blood glucose levels were determined in whole venous blood with an automatic glucose meter (Accu-check Aviva blood glucose meter, Roche Diagnostics, Tokyo, Japan). The blood pressure was measured by telemetry (Data Science Inc., MN55126, USA) before, during, and after MCAO-R and recorded using the Dataquest Advanced Research Technologies Acquisition program (Unique Medical).

\section{Measurement of the Infarct Volume}

The brains were extracted and equal 2-mm-spaced slices and six coronal blocks were prepared immediately using a brain matrix (Bioresearch Center, Nagoya, Japan). The samples did not contain olfactory tissue or tissue from the cerebellum. All but the 3 rd coronal sections were immersed in a 2,3,5-triphenyltetrazolium chloride (TTC) solution in PBS to detect the infarct area. We identified the area surrounding the infarct area in the frontal cortex as the peri-infarct area. The tissue samples were stored at $-80{ }^{\circ} \mathrm{C}$ until Western blot analysis and determination of the mRNA level. The extent of ischemic infarction was traced manually, and the integrated volume was calculated using NIH 1.36b Image J software. Artifacts from brain edema were eliminated by applying the indirect measurement method based on the contralateral brain volume.

\section{Neurological Assessment}

Neurological deficits were assessed by an examiner blinded to the treatment the rats had undergone. We modified the neurological scoring system of Huang et al. [17] and Chen et al. [18] and recorded our findings as $0=$ normal; $1=$ forelimb or hindlimb flexion, head turned $>10$ to the vertical axis within $30 \mathrm{~s}$ after raising the rat by the tail, inability to walk straight on the floor, and grasping the side of the beam during the beam balance test; 2 = circling toward the paretic side, one limb falling off the beam; $3=$ falling to the paretic side, hugging the beam, and two limbs falling off the beam; $4=$ attempting to balance on the beam but falling off ( $>40 \mathrm{~s}) ; 5=$ attempting to keep balance on the beam but falling off ( $>20 \mathrm{~s})$; and $6=$ falling off the beam without attempting to balance or hang on to the beam $(<20 \mathrm{~s})$. The rats were evaluated immediately after successful MCAO, $24 \mathrm{~h}$ after MCAO-R, and again on days 1,3 , and 7 (males) and on days 1, 3, 7, and 14 (OVX/ $\mathrm{GFP}^{+\mathrm{BM}}$ rats) after treatment with PGZ or VC. The total maximum score was 12 .

\section{Quantitative Real-Time PCR}

Total RNA obtained from the peri-infarct area was isolated with the BioRobot EZ1 and EZ1 universal tissue kit (Qiagen, Tokyo, Japan). RNA was converted to cDNA using the transcript first-strand cDNA synthesis kit (Qiagen). Quantitative real-time PCR assay of each sample was on Light Cycler FastStart DNA Master SYBR Green I and Roche LightCycler 2.0 (Roche Diagnostics, Tokyo, Japan) instruments. Primers for GAPDH were from Roche and used according to the manufacturer's directions. The other primers were: for rat IL-6, forward (F), 5'-TCT CAG GGA GAT CTT GGA AAT G-3', reverse (R), 5'-TAG AAA CGG AAC TCC AGA AGA C-3'; for rat TNF- $\alpha$, (F), 5'-CCC AAC AAG GAG GAG AAG T-3', (R), 5'-CGC TTG GTG GTT TGC TAC-3'; for rat IL-1 $\beta,(\mathrm{F}), 5^{\prime}$-TGC AGG CTT CGA GAT GAA C-3' (R), 5'-AGC TCA TGG AGA ATA CCA CTT 
G-3'; for rat VEGF, (F), 5'-CACATAGGAGAGATGAGCTT3', (R), 5'-CTGGCTTTG TTCTATCTTTC-3'. The amplified product was separated on $1.5 \%$ agarose gels containing $\mathrm{EtBr}$ solution (Wako, Osaka, Japan) and visualized on an ultraviolet transilluminator. The results were normalized to the expression of GAPDH mRNA.

\section{Immunohistochemistry}

The rats were transcardially perfused with $4 \%$ paraformaldehyde in PBS on ice. Their brains were fixed and 6- $\mu$ m-thick frozen sections were mounted on Matsunami adhesive silane (MAS)-coated glass slides (Matsunami Glass, Tokyo, Japan), blocked with serum-free protein (DakoCytomation), and then the slides were incubated with primary antibodies diluted with Canget signal immunostain (Toyobo, Osaka, Japan). The antibodies were rabbit polyclonal antibody against PPAR $\gamma$ (Abcam, Tokyo, Japan), GFAP, Nestin, MAP2, (Santa Cruz Biotechnology), and antibodies against GFP (Cell Signaling Technology). We used mouse monoclonal antibody against neuronal nucleus (NeuN) (Millipore, Tokyo, Japan), Musashi-1 (AbD Serotec), CD16, CD68 (Santa Cruz Biotechnology), CD31 (MAB1393, CHEMICON, MA), caspase-3, GFP, and Nestin (Cell Signaling Technology). The tissue samples were mounted with Vectashield (Vector Laboratories Inc., Burlingame, CA). Visualization was with Alexa Fluor 594 donkey anti-rabbit IgG or 488 goat antimouse IgG (Molecular Probes, Eugene, OR); the slides were examined under a fluorescence microscope (KEYENCE, BZX710, Osaka, Japan). To examine the specificity of immunoreactivity, the primary antibody was omitted to provide a nonspecific control. A parallel set of tissue sections was subjected to hematoxylin and eosin staining to identify the infarct core and the peri-infarct region. We counted all cells positive for Musashi-1 and GFP in the peri-infarct area. Images were captured at $\times 10$ magnification under the microscope. In each animal, we randomly selected two areas containing positive cells in $150 \times 150-\mu \mathrm{m}$ fields around the peri-infarct area. Tissue samples from four rats in each group were analyzed by densitometry of the positive cells using BZH-3A (KEYENCE) analysis software.

\section{Bromodeoxyuridine (BrdU) Labeling in OVX/GFP ${ }^{+B M}$ Rats}

We recorded the time course of proliferating cells in the brain after cerebral ischemia by pulse-labeling. BrdU, a thymidine analogue that is incorporated into the DNA of dividing cells during the S-phase, was used for mitotic labeling (Sigma Chemical) [19]. We applied a cumulative labeling method to examine the population of proliferating cells in OVX rats exposed for 14 days to cerebral ischemia and injected BrdU $(50 \mathrm{mg} / \mathrm{kg}$, i.p.) every $4 \mathrm{~h}$ for $12 \mathrm{~h}$ before killing the rats on the $1 \mathrm{st}, 3 \mathrm{rd}, 7 \mathrm{th}$, or 14 th day after the induction of cerebral ischemia ( $n=6$ each). BrdU-positive cells were detected immunohistochemically using sheep polyclonal antibody BrdU (LifeSpan BioSciences Inc.) as the primary and donkey anti-sheep IgG (Molecular Probes, Eugene, OR) as the secondary antibody.

\section{Western Blot Analysis in OVX/GFP ${ }^{+B M}$ Rats}

Brain tissue in the peri-infarct area was homogenized and sonicated in RIPA buffer (Thermo Scientific, Rockford, IL) containing phosphatase and protease inhibitors (Roche, Tokyo, Japan) and centrifuged. Total protein in the supernatant was measured with the BCA protein assay kit (Pierce, Rockford, IL). Protein was separated by 7.5 or $12 \%$ SDSPAGE and transferred to a polyvinylidenedifluoride membrane. After blocking with $5 \%$ skim milk or BSA in Trisbuffered saline solution-Tween 20 (T-TBS), the membrane was incubated with the primary antibodies in Canget signal immunostain or T-TBS. The same primary antibodies as used for the immunohistochemical studies, rabbit polyclonal antibody against p-Akt (Cell Signaling Technology), MAP2, and mouse monoclonal anti- $\beta$-actin (Sigma, Tokyo Japan) were used. After incubation with horseradish peroxidaseconjugated secondary antibodies (GE Healthcare, Buckinghamshire, UK), signals were detected by chemiluminescence using an ECL-plus kit (GE Healthcare). Images were analyzed with Image Quant LAS 4000 mini (GE Healthcare) and Image $\mathrm{J}$ software and quantified as the relative increase over the controls after normalization with $\beta$-actin.

\section{Statistical Analysis}

Power estimates were calculated based on $\alpha=0.05$, $1-\beta=0.8$, and a surgery-related drop-out rate of around $10 \%$ to obtain group sizes appropriate for detecting an effect size of 0.4 based on a preliminary experiment using $\mathrm{G}^{*}$ Power 3.1. The infarct volume and the neurological score were analyzed with Student's $t$ test and ManWhitney $U$ test, respectively; their correlation was assessed by the Spearman's rank-correlation coefficient. The mRNA expression levels were determined with analysis of variance (ANOVA) followed by Scheffe's test for three-group comparisons. Statistical analyses were performed using IBM SPSS Statistics 22. Data are shown as the mean \pm SD. Differences were considered statistically significant at $p<0.05$. 
Fig. 1 Effects of PGZ against brain damage in male rats. Postischemia treatment with PGZ or vehicle was performed immediately after MCAO induction on day 0 and once a day for 7 consecutive days. The neurological scores (a), body weight (b), and infarct volume (c) were recorded in PGZ- and vehicle-treated male rats. The infarct volume was recorded as a percentage of the contralateral hemisphere using Image $\mathrm{J}$ software (each group $n=12$ ). The correlation between the infarct volume and neurological deficits was assessed by Spearman's rank-correlation coefficient (d). Each bar represents the mean \pm SD. $* p<0.05$ vs. VC by ANOVA followed by Scheffe's test. MCAO-R middle cerebral artery occlusion-reperfusion, VC vehicle control, PGZ $2.5 \mathrm{mg} / \mathrm{kg}$ pioglitazone
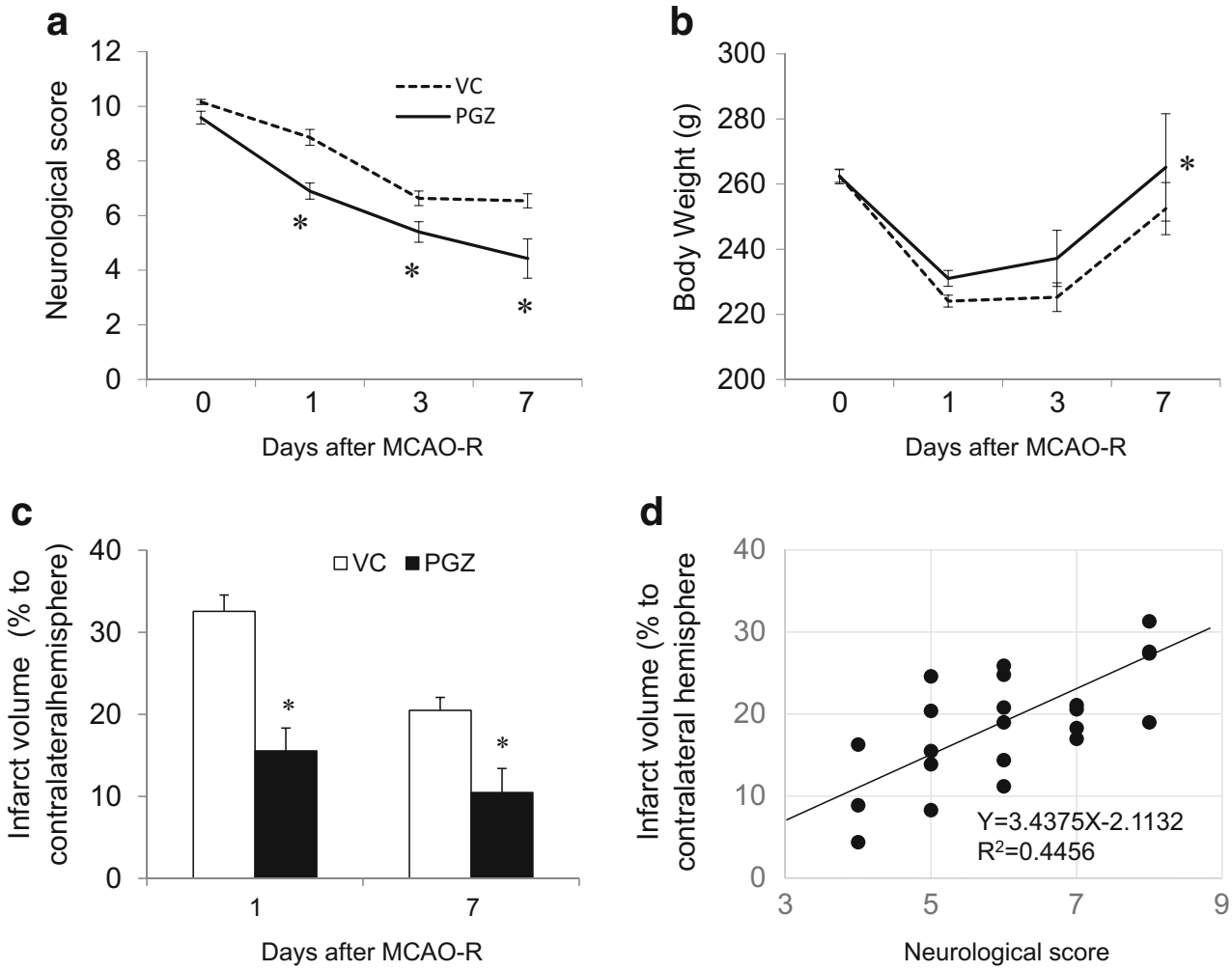

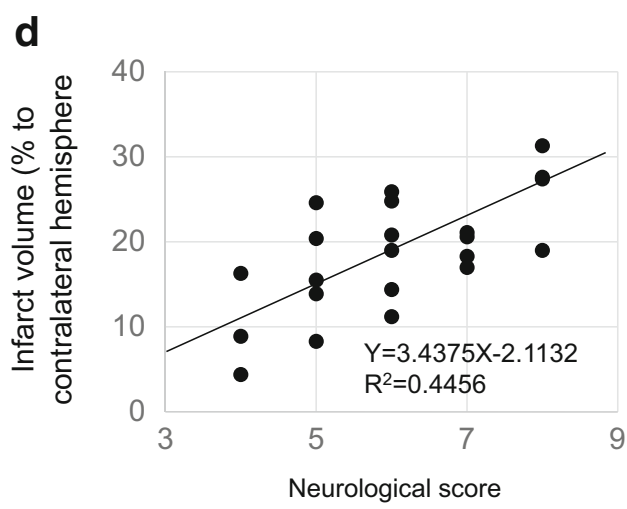

\section{Results}

Male Rats: Treatment with PGZ in the Early Post-ischemia Phase Reduced the Cerebral Infarct Size and Ameliorated Neurological Deficits by Inhibiting Pro-inflammatory Responses

We first assessed the effects of PGZ treatment in the early phase after experimental cerebral ischemia. Compared to the VC males, on days 1-7 after the ischemic insult, PGZ rats manifested a lower neurological score and earlier recovery of the body weight loss (Fig. 1a, b). The infarct volume was smaller than in VC rats (Fig. 1c) and correlated with the neurological score (Fig. 1d). Next, to address the mechanisms underlying the effects of PGZ, we examined its antiinflammatory effects against brain ischemic injury. In VC rats, the mRNA level of IL- 6 , IL- $\beta$, and TNF $\alpha$ was increased $3 \mathrm{~h}$ after ischemia induction and augmented at $24 \mathrm{~h}$ (Fig. $2 \mathrm{a}-\mathrm{c}$ ). In PGZ rats, the mRNA level of IL-6 (Fig. 2a) but not of IL- $\beta$ and $\mathrm{TNF} \alpha$ was significantly decreased at 3 and $24 \mathrm{~h}$ (Fig. 2b, c). Immunohistochemically, the expression of PPAR $\gamma$ was higher in PGZ than VC rats; CD16- and CD68-positive cells were fewer and the expression of caspase-3 was lower (Fig. 2d). PPAR $\gamma$ was localized in CD31-positive cells. CD16-positive cells were Iba-1 or CD68 positive. Treatment with PGZ in the early phase post-ischemia appeared to exert beneficial effects through anti-apoptosis and antiinflammatory response effects elicited by the expression of $\operatorname{PPAR} \gamma$.

\section{OVX/GFP ${ }^{+B M}$ Rats: Increased PPAR $\gamma$ Expression Elicited by PGZ Was Associated with Neuroprotection and the Activation of Resident Stem Cells in the SVZ and the Recruitment of BM Cells}

Our earlier study in ovariectomized (OVX) rats treated with PGZ before ischemia induction suggested neurogenesis in the post-ischemic phase [8]. To examine the role in neuroprotection and neurogenesis of early-phase PGZ treatment after ischemia induction, we used $\mathrm{OVX} / \mathrm{GFP}^{+\mathrm{BM}}$ rats whose $\mathrm{BM}$ cells were replaced by $\mathrm{BM}$ cells from GFP rats. While $75 \%$ of the $\mathrm{VC}-\mathrm{OVX} / \mathrm{GFP}^{+\mathrm{BM}}$ rats died within 14 days after MCAO-R, all OVX/GFP ${ }^{+B M}$ rats treated with PGZ (PGZ rats) survived ( $n=12$ in each group). On days $1,3,7$, and 14 after MCAO-R, the cerebral infarct volume was significantly smaller in PGZ than VC rats $(p<0.05$, Fig. 3a) and their neurological deficits were significantly less pronounced ( $p<0.05$, Fig. 3b), indicating neuroprotection by earlyphase PGZ treatment. There was no significant difference in the cerebral blood flow, blood glucose levels, and blood pressure between the PGZ and VC rats during and after MCAO-R (data not shown). 

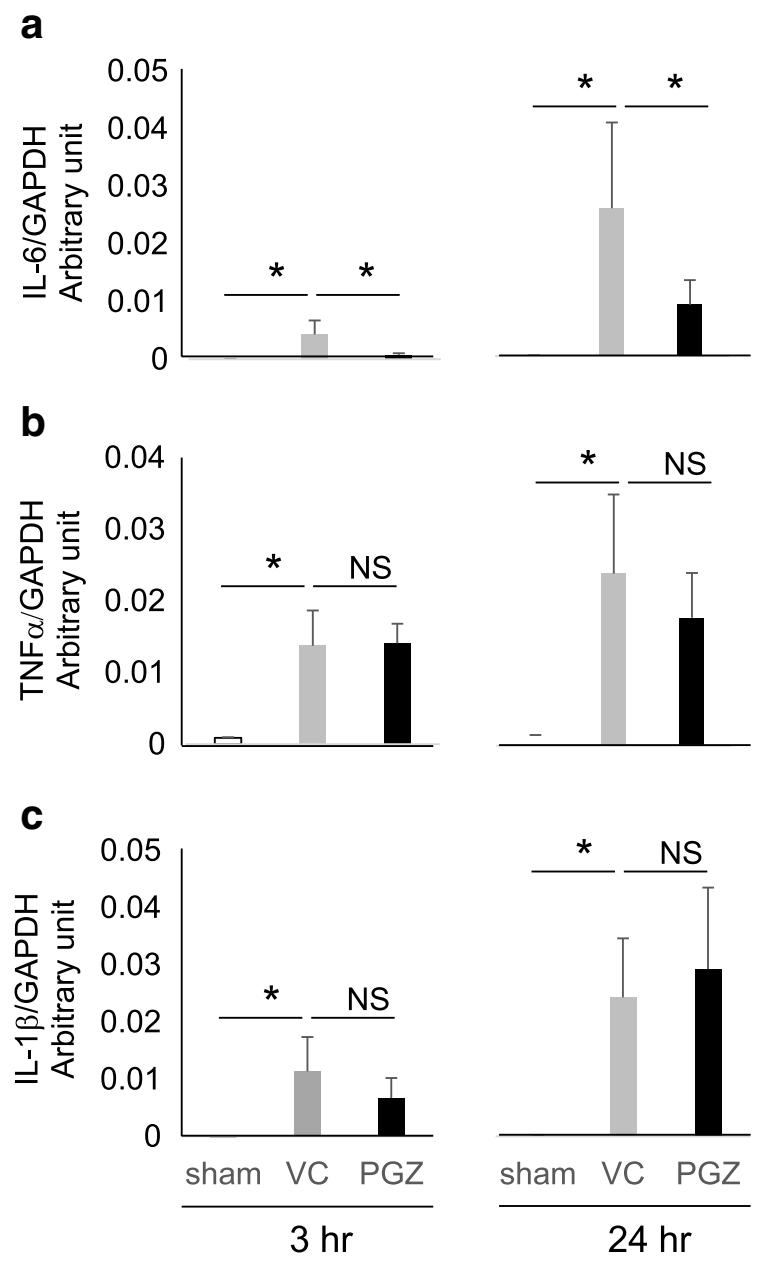

Fig. 2 The mRNA level of pro-inflammatory cytokines and representative immunohistochemistry findings in PGZ- and VC-treated male rats. The mRNA level of the pro-inflammatory cytokines IL-6 (a), TNF $\alpha$ (b), and IL-1 $\beta$ (c) was assessed by quantitative real-time PCR assay and normalized by GAPDH. Data obtained 3 and $24 \mathrm{~h}$ after MCAO-R are shown. Data are the mean $\pm \mathrm{SD}$ from eight rats per group. ${ }^{*} p<0.05 \mathrm{vs.}$

Compared to the contralateral non-ischemic side of $\mathrm{VC}$ rats, on days 3 and 7 post-MCAO-R, the expression of PPAR $\gamma$ and of Musashi-1- and GFP-positive cells was increased in the SVZ on the infarct side of PGZ rats (Fig. 3c). The expression of PPAR $\gamma$ in the SVZ seemed to be associated with an increase in innate stem cells and the recruitment of allogeneic BM-derived stem cells.

\section{OVX/GFP ${ }^{+B M}$ Rats: PPAR $\gamma$ Activation Promoted the Proliferation of Stem Cells in the SVZ and their Translocation to the Peri-infarct Region}

As shown in Fig. 4a, on the 7th day after ischemia induction, nestin-positive cells were detected in the SVZ and the cortical peri-infarct area of PGZ rats; the cells seemed to migrate from
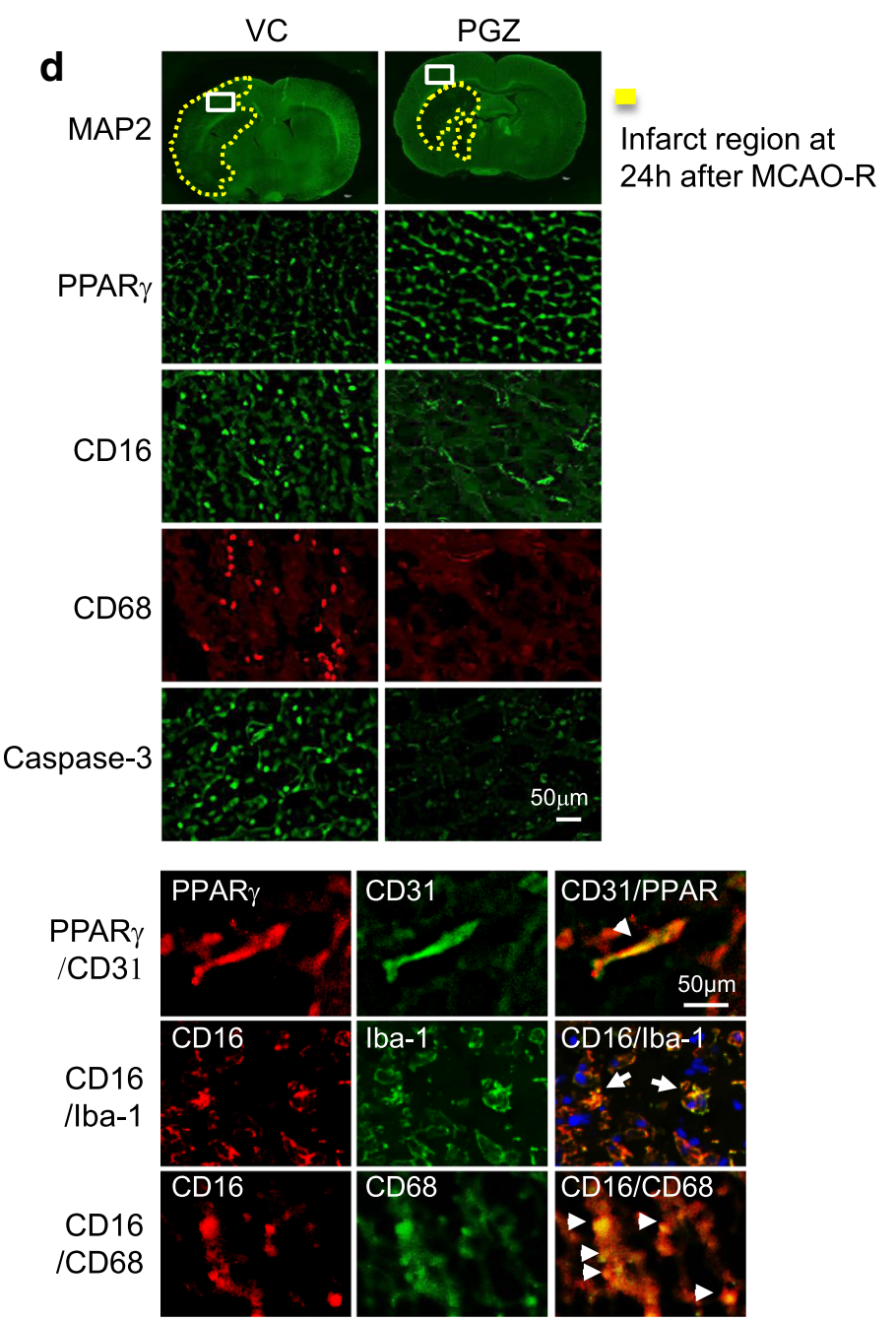

VC by ANOVA followed by Scheffe's test. The expression of PPAR $\gamma$, CD16, CD68, and caspase-3 was examined in the peri-infarct region of PGZ-treated and VC rats $24 \mathrm{~h}$ after MCAO (d). PPAR $\gamma$ - and CD16positive cells were co-localized with VEGF-, Iba-1- or CD68-positive cells

the SVZ to the peri-infarct region. The number of translocated Musashi-1-positive- and of GFP-positive cells was significantly higher in PGZ than VC rats $(p<0.05$, Fig. $4 b$ ) and associated with the increase in NeuN- and GFAP-positive cells. These cell populations seemed to include both resident stem cells in SVZ and recruitment of BM-derived stem cells replaced by allogeneic BM cells. We immunohistochemically assessed the incorporation of the cell proliferation marker BrdU in the stem cells. The presence of many BrdU-labeled Musashi-1- and GFP-positive cells was evidence for the proliferation of stem cells in the peri-infarct region (Fig. 4c). PPAR $\gamma$ activation may promote not only the proliferation of stem cells in the SVZ but also the migration of proliferated stem cells from the SVZ to the cortical periinfarct region. 


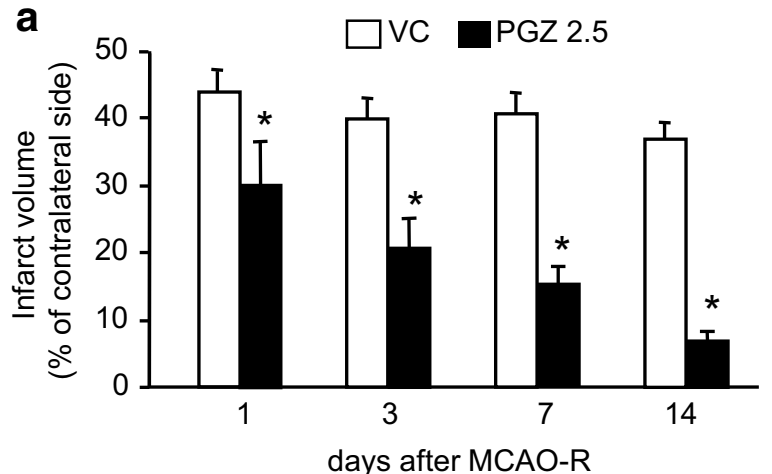

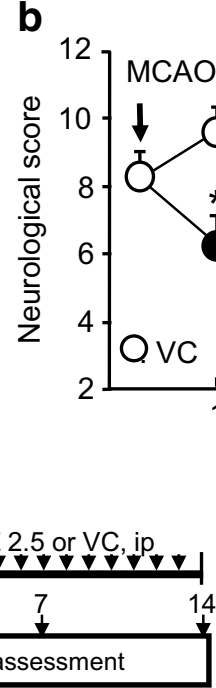

C

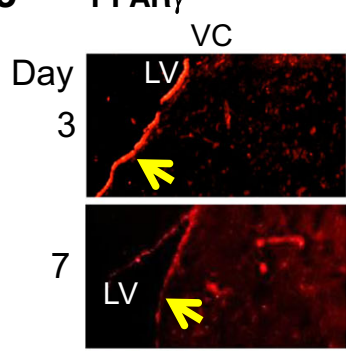

14

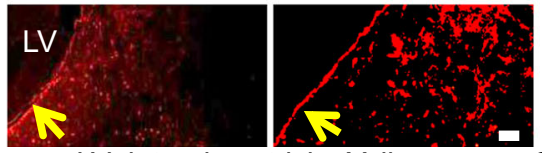

Musashi-1
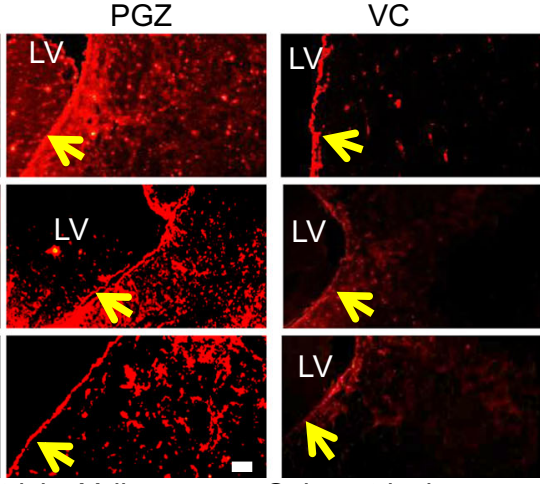

LV; lateral ventricle, Yellow arrow; Subventricular zone, Bars $=100 \mu \mathrm{m}$
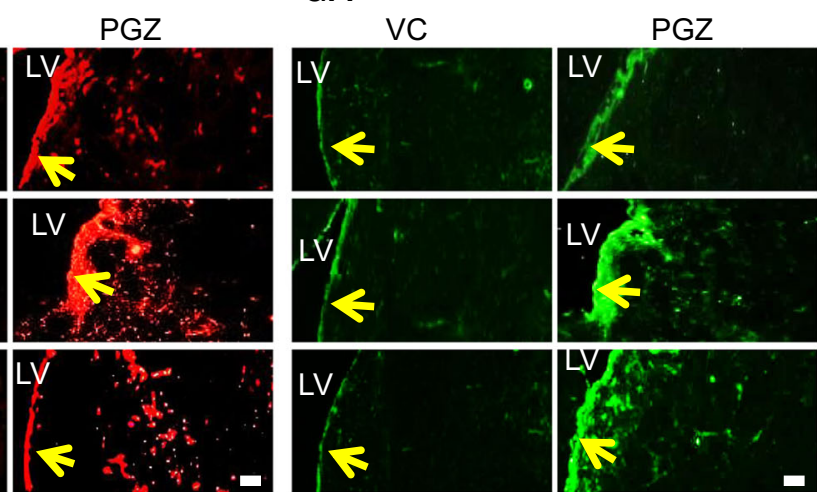

Fig. 3 Effects of post-ischemia treatment with $\mathrm{PGZ}$ in OVX/GFP ${ }^{+\mathrm{BM}}$ rats. PGZ or vehicle (VC) was administered immediately after MCAO induction on day 0 and once a day for 14 consecutive days. The infarct volume (a) was recorded in OVX/GFP ${ }^{+\mathrm{BM}}$ rats treated with $\mathrm{PGZ}$ or VC (each group $n=12$ ). The neurological score (b) was assessed as described in Materials and Methods. Each bar represents the mean \pm SD $* p<0.05$ vs. VC by ANOVA followed by Scheffe's test. Representative immunohistochemistry findings for PPAR $\gamma$, Musashi-1, and GFP (d) in the SVZ after MCAO-R

mRNA level of VEGF was increased in the peri-infarct region ( $p<0.05$, Fig. 5d). Consequently, the expression of VEGF, MAP2, and p-Akt was significantly higher in PGZ than VC rats $(p<0.05$, Fig. 5e). These findings suggest that the upregulation by PGZ of survival signaling pathways was associated with the proliferation of neuronal stem cells and their differentiation into mature neurons, glia, and blood vessels.

\section{Discussion}

We first demonstrate that post-ischemia treatment with PGZ in early phase prevented the expansion of brain injury and attenuated neurological deficits in male and $\mathrm{OVX} / \mathrm{GFP}^{+\mathrm{BM}}$ rats. We showed that the neuroprotective effects of PGZ in the early post-ischemia phase were associated with an anti- 
a

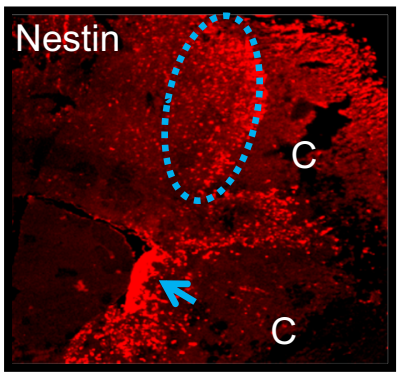

${ }_{0} ;$ cortical peri-infarct region

R; SVZ

C; Infact core

at day 7 b

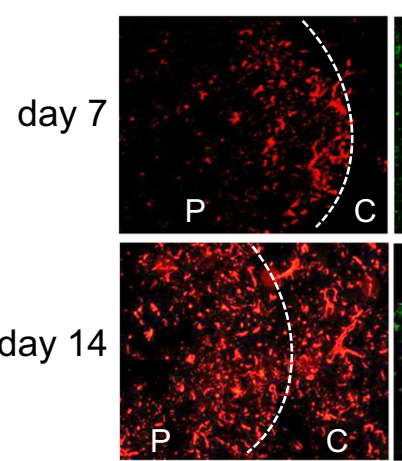

P; Peri-infarct region
GFP
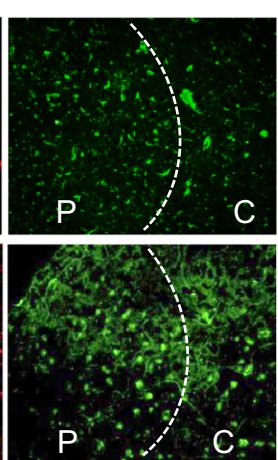

C; Infarct core
NeuN

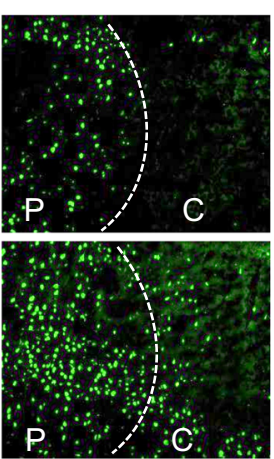

$P$

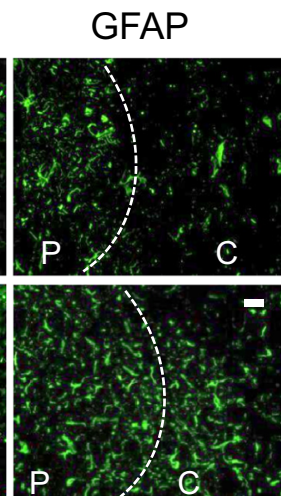

Bar $=100 \mu \mathrm{m}$

C
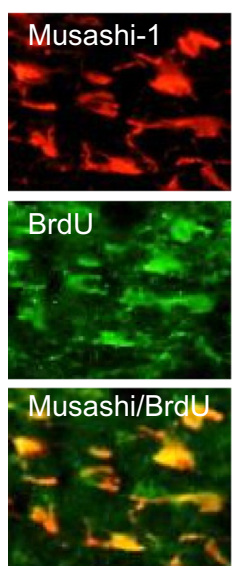
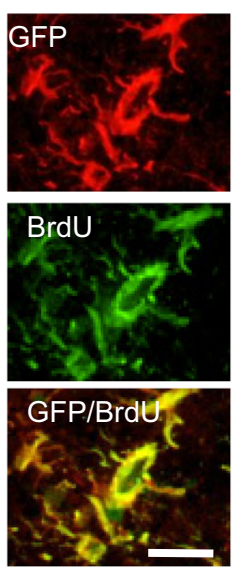

Bar $=50 \mu \mathrm{m}$
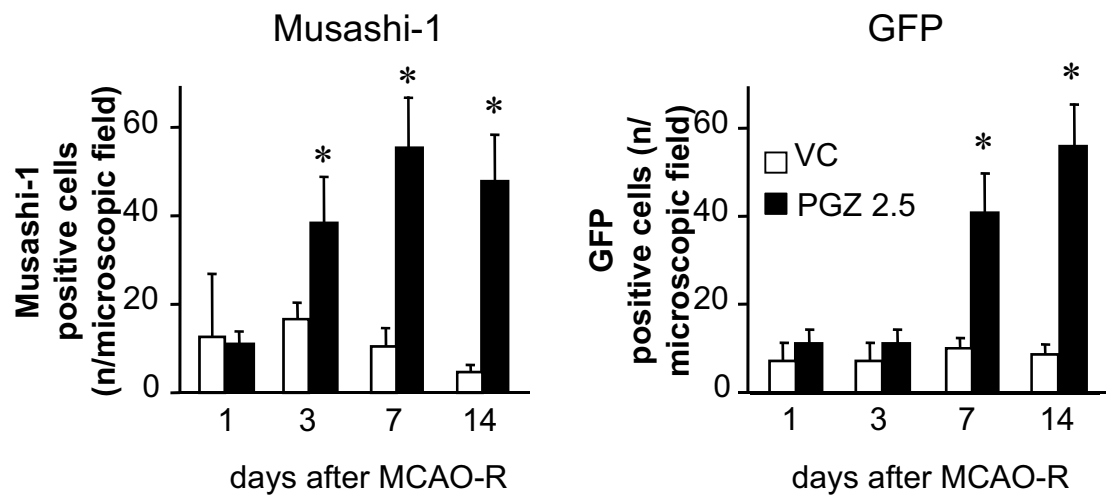

Fig. 4 Stem cells migration from the SVZ into the cortical peri-infarct region promoted by PGZ in OVX/GFP ${ }^{+\mathrm{BM}}$ rats. Representative nestinpositive cells in the SVZ and the cortical peri-infarct region on day 7 after ischemia induction in $\mathrm{PGZ}$-treated $\mathrm{OVX} / \mathrm{GFP}^{+\mathrm{BM}}$ rats (a). Cells positive for Musashi-1, GFP, NeuN, and GFAP were detected in the infarct- and the peri-infarct area (b). Musashi-1- and GFP-positive cells were counted in $150 \times 150-\mu \mathrm{m}$ fields. The total cell number $(n)$ in each area was 140 160 cells for DAPI. Each bar represents the mean \pm SD from two areas in each of four rats. $* p<0.05 \mathrm{vs}$. VC by ANOVA followed by Scheffe's test. Representative Musashi-1- and GFP-positive cells incorporated BrdU (c) inflammatory response involving a decrease in IL-6 and M1type macrophages in male rats. Furthermore, we found that in $\mathrm{OVX} / \mathrm{GFP}^{+\mathrm{BM}}$ rats $\mathrm{PGZ}$ promoted the proliferation of both innate stem cells in the SVZ and BM-derived stem cells and their translocation from the SVZ into the cortical peri-infarct region on days 7-14 of post-ischemia. This was associated with the high expression of VEGF, MAP2, and p-AKT and an increase in PPAR $\gamma$ in the peri-infarct area and the differentiation of stem cells into mature cells, resulting in the alleviation of neurological dysfunction. Our findings suggest that early-phase treatment with PGZ, even after experimentally induced cerebral ischemia, contributed to a good stroke outcome in rats via its anti-inflammatory effects and the elicitation of neurogenesis (Fig. 6).

Lambertsen et al. [20] who studied experimental and human stroke, reported that within $1-6 \mathrm{~h}$ post-ischemia, the protein level of IL- $1 \beta$ and TNF $\alpha$ but not of IL- 6 was increased in the brain and cerebrospinal fluid and persisted at high levels until $24 \mathrm{~h}$. In our animal study, the mRNA level of IL-6, TNF $\alpha$, and IL- $1 \beta$ was increased $24 \mathrm{~h}$ after ischemia induction; this was associated with the presence of M1-like macrophages and the expansion of cerebral infarction although the mRNA level of IL- 6 was low during the first $3 \mathrm{~h}$. PGZ suppressed the expression of IL-6 at $24 \mathrm{~h}$ without affecting TNF $\alpha$ and IL-1 $\beta$. During the early post-ischemic phase in male rats, the different effects of PGZ against these molecules may be associated with their different expression profile after ischemia and the timing of PGZ treatment. Nakashiro [10] reported that PGZ decreased the expression of circulating inflammatory monocytes. As PGZ inhibited the expression of $\mathrm{CD} 16^{+} / \mathrm{CD} 68^{+} \mathrm{M} 1-$ like macrophages in our rats, we think that in the early phase of post-ischemia, it inhibited the recruitment 

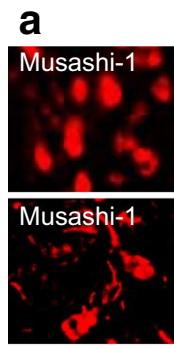

Musashi-1

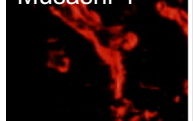

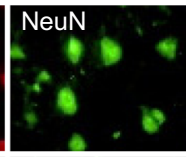

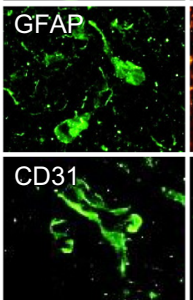

b

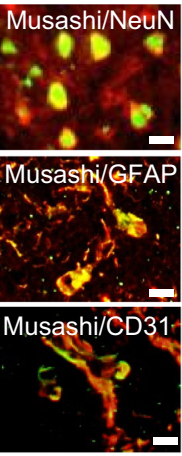

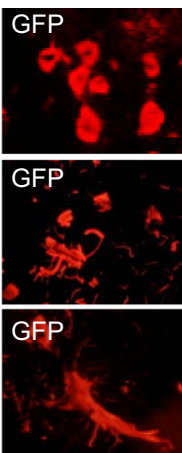

C

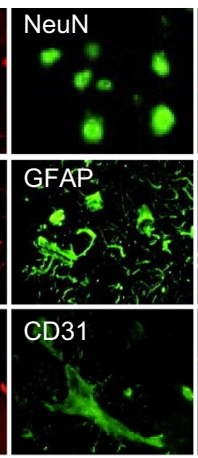

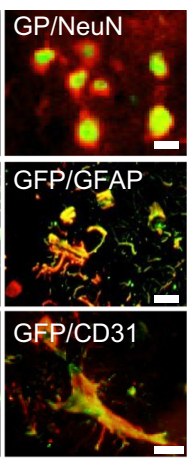

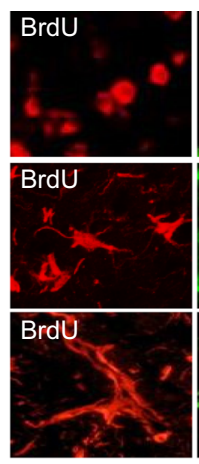

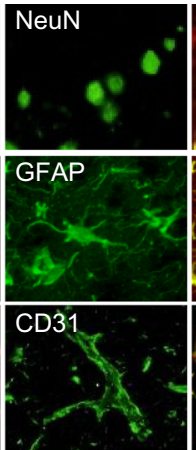

BrdU/NeuN

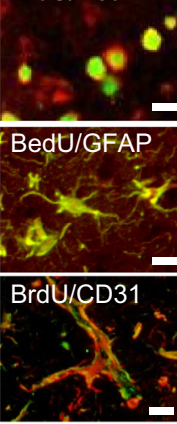

Bars $=50 \mu \mathrm{m}$

d

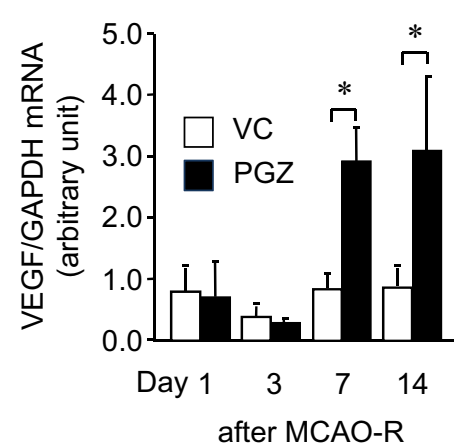

e

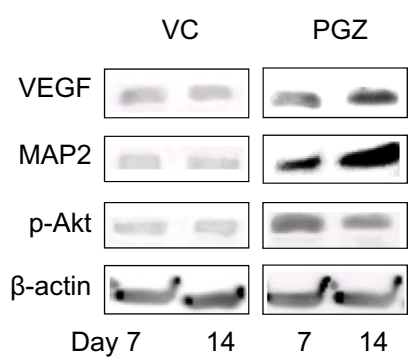

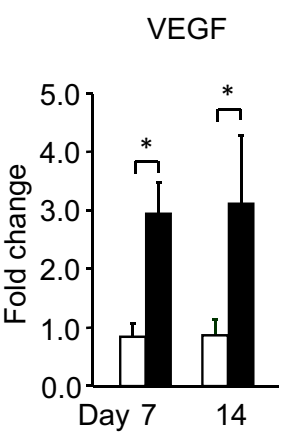

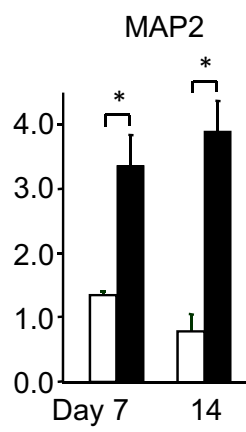

p-Akt

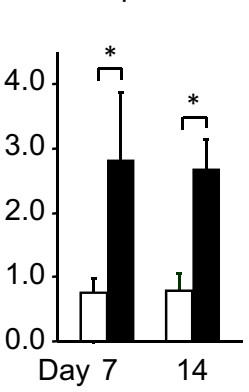

Fig. 5 Stem cells differentiation into blood vessels, neurons, and glia via the upregulation of the survival signal pathway induced by PGZ in OVX/ $\mathrm{GFP}^{+\mathrm{BM}}$ rats. In the peri-infarct region, double immunohistochemical staining for Musashi-1 (a), GFP (b), and BrdU (c) shows colocalization with NeuN, GFAP, and CD31, respectively. The mRNA level

of M1-like monocyte-derived macrophages from the perivascular area into the brain. According to Patzer et al. [21], IL-6-expressing microglia/macrophages in the brain were activated in the initial post-ischemic stage and immunohistochemical and Western blot analysis showed that PGZ reduced the expression of IL-6. Thus, the down-regulation of IL-6 in the early post-ischemic phase may be crucial for a reduction in ischemic brain damage.

Although we do not know how PGZ increases the expression of PPAR $\gamma$ in areas adjacent to the SVZ, elsewhere [8], we demonstrated that treatment with PGZ before ischemia induction upregulated STAT3 and increased the expression of antiapoptotic bcl-2 and VEGF. IL-6 is associated with the JAK/ STAT pathway and elicits a pro-inflammatory response in the early phase; in the late phase, it is associated with neurotrophic effects [22]. The regulation of IL-6 by PGZ may exert ambivalent beneficial effects in the early and late phase after stroke.

Neuronal stem cells are primordial, multipotent, selfrenewing cells that give rise to differentiated progeny within all neuronal and glial lineages. They continue to produce new neurons throughout life in the SVZ and the dentate gyrus of the hippocampus $[19,23]$. However, most of the newly of VEGF (d) was analyzed by quantitative PCR. The protein expression of VEGF, p-Akt, and MAP2 in the peri-infarct region (e) was detected on Western blots and analyzed using LAS 4000 and Image J software. Data are the mean \pm SD from eight rats per group. $* p<0.05$ vs. VC by ANOVA followed by Scheffe's test

formed cells promptly undergo apoptosis, possibly due to unfavorable conditions after cerebral ischemia and the lack of adequate trophic support [6]. In contrast, PGZ-induced stem cells seem to be less affected by ischemic conditions. The increased expression of MAP2 and p-Akt elicited by PGZ and the upregulation of anti-apoptotic genes [8] might contribute to the activation of survival signaling pathways to nourish the stem cells. Thus, the activation of PPAR $\gamma$ by PGZ after the ischemic insult may not only accelerate the proliferation of resident stem cells in the SVZ and the recruitment of BMderived stem cells but may also contribute to their differentiation in the peri-infarct area.

Neurotrophic factors regulate the survival, proliferation, and differentiation of cells in the central nervous system [6, 23]. VEGF, identified as an angiogenic and vascular permeability factor, is recognized as a neurotrophic factor [24] whose role depends on its temporal and spatial profiles. During the acute phase of ischemic injury, the upregulation of VEGF in cerebral vessels increases the permeability of the blood-brain-barrier, thereby exacerbating ischemic cell damage [25]. At a later stage after stroke, it triggers angiogenesis, promotes the blood supply to the brain, and accelerates 


\section{Schematic role of PPAR $\gamma$ activation on post-stroke}

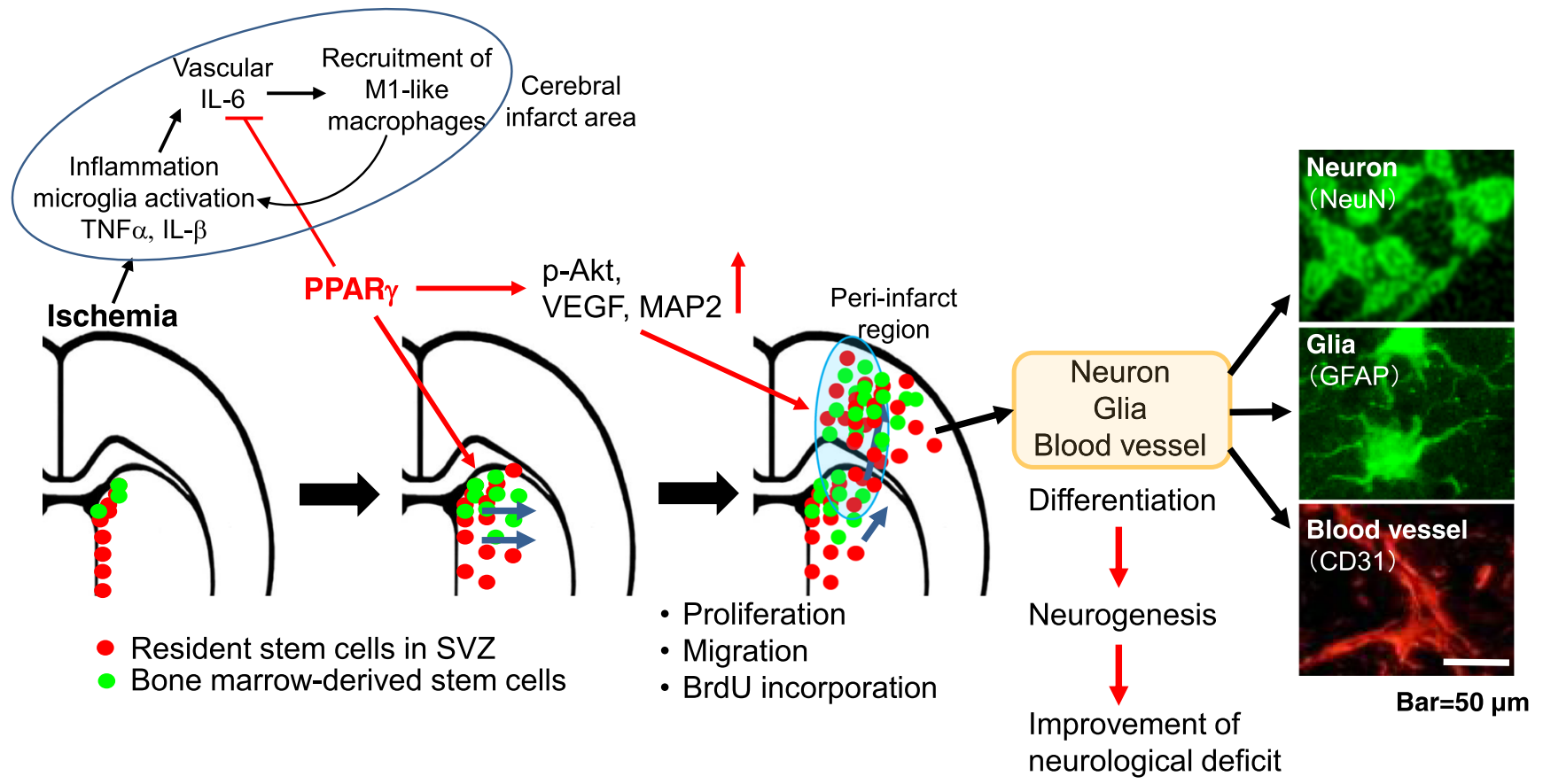

Fig. 6 Schematic role of PGZ administered in the early phase after cerebral ischemia. Activation of PPAR $\gamma$ by PGZ inhibits proinflammatory responses and promotes neurogenesis in the peri-infarct

neurogenesis [26]. Despite the high expression of PPAR $\gamma$ elicited by PGZ in both the early and late phase after the ischemic insult imposed on our rats, the VEGF expression pattern was different in these phases. The upregulation of VEGF on days 7 and 14 may have contributed to angiogenesis. The phosphatidylinositol-3 kinase (PI3)/Akt signaling pathway plays a central role in regulating the growth, proliferation, and survival of cells under physiological and pathophysiological conditions. Its activation protects vascular function [27], promotes cell survival, and suppresses apoptosis. As did others $[28,29]$, we found that treatment with PGZ even after an ischemic insult increased the expression of p-Akt and MAP2 in the peri-infarct region. Therefore, elicitation of angiogenesis and neurogenesis may be attributable to the upregulation of survival signaling pathways by PGZ.

Although attempts to promote neurogenesis by injecting exogenous stem cells have remained unsuccessful, the combined delivery of PGZ and exogenous stem cells may help to promote neurogenesis. We need further studies to determine whether PGZ helps to promote the survival and proliferation of exogenous stem cells. Kernan et al. [30] reported that 4.8year PGZ treatment of patients with insulin resistance, ischemic stroke, and transient ischemic attacks reduced the risk for recurrent stroke or myocardial infarcts but increased the risk for weight gain, edema, and bone fracture. Therefore, the condition of patients treated with PGZ must be monitored carefully, and its administration should be short term. region. Some NeuN (green)-, GFAP (green)-, or CD31 (red)-positive cells were mature neurons, extended axons, glias, and blood vessels

In conclusion, we provide new evidence that PGZ treatment in the early phase after an experimentally induced ischemic insult ameliorated neurological dysfunction and suppressed the infarct size in male and $\mathrm{OVX} / \mathrm{GFP}^{+\mathrm{BM}}$ rats. We show that its beneficial effects were associated with the induction of antiinflammatory responses in the early and with the elicitation of angiogenesis and neurogenesis in the late post-ischemic phase. Treatment with PGZ in the early post-ischemic phase may help to limit ischemic brain damage and to alleviate neuronal deficits. We are continuing to assess the potential role of PGZ in the activation of post-stroke PPAR $\gamma$ because such findings may lead to treatments that improve the outcomes in stroke patients.

Funding This work was supported by a Grant-in-Aid for Scientific Research [JSPS KAKENHI Grant Number JP15K10306] and a Grant-inAid for the Strategic Young Researcher Overseas Visits Program for Accelerating Brain Circulation from the Japan Society for the Promotion of Science [JSPS Grant Number JPS2407].

\section{Compliance with Ethical Standards}

Ethical Approval Our animal study was approved by the Ethics Committee of the Institute of Biological Medicine of the University of Tokushima Graduate School and followed all applicable international, national, and/or institutional guidelines for the care and use of animals.

Conflict of Interest The authors declare that they have no conflicts of interest. 
Open Access This article is distributed under the terms of the Creative Commons Attribution 4.0 International License (http:// creativecommons.org/licenses/by/4.0/), which permits unrestricted use, distribution, and reproduction in any medium, provided you give appropriate credit to the original author(s) and the source, provide a link to the Creative Commons license, and indicate if changes were made.

\section{References}

1. Feigin VL, Lawes CM, Bennett DA, Anderson CS. Stroke epidemiology: a review of population-based studies of incidence, prevalence, and case-fatality in the late 20th century. Lancet Neurol. 2003;2(1):43-53.

2. Bao X, Wei J, Feng M, Lu S, Li G, Dou W, et al. Transplantation of human bone marrow-derived mesenchymal stem cells promotes behavioral recovery and endogenous neurogenesis after cerebral ischemia in rats. Brain Res. 2011;7:103-13. https://doi.org/10. 1016/j.brainres.2010.10.063.

3. Emsley JG, Mitchell BD, Kempermann G, Macklis JD. Adult neurogenesis and repair of the adult CNS with neural progenitors, precursors, and stem cells. Prog Neurobiol. 2005;75(5):321-41. https://doi.org/10.1016/j.pneurobio.2005.04.002.

4. Kornack DR, Rakic P. The generation, migration, and differentiation of olfactory neurons in the adult primate brain. PNAS (USA). 2001;98(8):4752-7. https://doi.org/10.1073/pnas.081074998.

5. Bernier PJ, Vinet J, Cossette M, Parent A. Characterization of the subventricular zone of the adult human brain: evidence for the involvement of Bcl-2. Neurosci Res. 2000;37(1):67-78.

6. Arvidsson A, Collin T, Kirik D, Kokaia Z, Lindvall O. Neuronal replacement from endogenous precursors in the adult brain after stroke. Nat Med. 2002;8(9):963-70. https://doi.org/10.1038/nm747.

7. Tureyen K, Kapadia R, Bowen KK, Satriotomo I, Liang J, Feinstein DL, et al. Peroxisome proliferator-activated receptor-gamma agonists induce neuroprotection following transient focal ischemia in normotensive, normoglycemic as well as hypertensive and type-2 diabetic rodents. J Neurochem. 2007;101(1):41-56. https://doi.org/ 10.1111/j.1471-4159.2006.04376.x.

8. Kinouchi T, Kitazato KT, Shimada K, Yagi K, Tada Y, Matsushita N, et al. Activation of signal transducer and activator of transcription-3 by a peroxisome proliferator-activated receptor gamma agonist contributes to neuroprotection in the peri-infarct region after ischemia in oophorectomized rats. Stroke. 2012;43(2):478-83. https://doi.org/10. 1161/STROKEAHA.111.618926.

9. Sakai S, Sato K, Tabata Y, Kishi K. Local release of pioglitazone (a peroxisome proliferator-activated receptor $\gamma$ agonist) accelerates proliferation and remodeling phases of wound healing. Wound Repair Regen. 2016;24(1):57-64. https://doi.org/10.1111/wrr.12376.

10. Nakashiro S, Matoba T, Umezu R, Koga J, Tokutome M, Katsuki S, et al. Pioglitazone-incorporated nanoparticles prevent plaque destabilization and rupture by regulating monocyte/macrophage differentiation in ApoE-/- mice. Arterioscler Thromb Vasc Biol. 2016;36(3): 491-500. https://doi.org/10.1161/ATVBAHA.115.307057.

11. Wurmser AE, Palmer TD, Gage FH. Neuroscience: cellular interactions in the stem cell niche. Science. 2004:304(5675):1253-5. https://doi.org/10.1126/science.1099344.

12. Lapchak PA, Zhang JH, Noble-Haeusslein LJ. RIGOR guidelines: escalating STAIR and STEPS for effective translational research. Transl Stroke Res. 2013;4(3):279-85. https://doi.org/10.1007/ s12975-012-0209-2.

13. Landis SC, Amara SG, Asadullah K, Austin CP, Blumenstein R, Bradley EW, et al. A call for transparent reporting to optimize the predictive value of preclinical research. Nature. 2012;490(7419): 187-91. https://doi.org/10.1038/nature11556.

14. Sata M, Saiura A, Kunisato A, Tojo A, Okada S, Tokuhisa T, et al. Hematopoietic stem cells differentiate into vascular cells that participate in the pathogenesis of atherosclerosis. Nat Med. 2002;8(4):403-9. https://doi.org/10.1038/nm 0402-403.

15. Liu H, Uno M, Kitazato KT, Suzue A, Manabe S, Yamasaki H, et al. Peripheral oxidative biomarkers constitute a valuable indicator of the severity of oxidative brain damage in acute cerebral infarction. Brain Res. 2004;1025(1-2):43-50. https://doi.org/10.1016/j. brainres.2004.07.071.

16. Yagi K, Kitazato KT, Uno M, Tada Y, Kinouchi T, Shimada K, et al. Edaravone, a free radical scavenger, inhibits MMP-9-related brain hemorrhage in rats treated with tissue plasminogen activator. Stroke. 2009;40(2):626-31. https://doi.org/10.1161/ STROKEAHA.108.520262.

17. Huang A, Huang PL, Panahian N, Dalkara T, Fishman MC, Moskowitz MA. Effects of cerebral ischemia in mice deficient in neuronal nitric oxide synthase. Science. 1994;265(5180):1883-5.

18. Chen J, Sanberg PR, Li Y, Wang L, Lu M, Willing AE, et al. Intravenous administration of human umbilical cord blood reduces behavioral deficits after stroke in rats. Stroke. 2001;32(11):2682-8.

19. Zhang RL, Zhang ZG, Zhang L, Chopp M. Proliferation and differentiation of progenitor cells in the cortex and the subventricular zone in the adult rat after focal cerebral ischemia. Neuroscience. 2001;105(1):33-41.

20. Lambertsen KL, Biber K, Finsen B. Inflammatory cytokines in experimental and human stroke. J Cereb Blood Flow Metab. 2012;32(9):1677-98. https://doi.org/10.1038/jcbfm.2012.88.

21. Patzer A, Zhao Y, Stöck I, Gohlke P, Herdegen T, Culman J. Peroxisome proliferator-activated receptors gamma (PPAR gamma) differently modulate the interleukin- 6 expression in the periinfarct cortical tissue in the acute and delayed phases of cerebral ischaemia. Eur J Neurosci. 2008;28(9):1786-94. https://doi.org/10. 1111/j.1460-9568.2008.06478.x.

22. Suzuki S, Tanaka K, Suzuki N. Ambivalent aspects of interleukin-6 in cerebral ischemia: inflammatory versus neurotrophic aspects. J Cereb Blood Flow Metab. 2009;29(3):464-79. https://doi.org/10. 1038/jcbfm.2008.141.

23. Gage FH. Mammalian neural stem cells. Science. 2000;287(5457): 1433-8.

24. Sun Y, Jin K, Xie L, Childs J, Mao XO, Logvinova A, et al. VEGFinduced neuroprotection, neurogenesis, and angiogenesis after focal cerebral ischemia. J Clin Invest. 2003;111(12):1843-51. https://doi. org/10.1172/JCI17977.

25. Ma Y, Zechariah A, Qu Y, Hermann DM. Effects of vascular endothelial growth factor in ischemic stroke. J Neurosci Res. 2012;90(10):1873-82. https://doi.org/10.1002/jnr.23088.

26. Kaya D, Gürsoy-Ozdemir Y, Yemisci M, Tuncer N, Aktan S, Dalkara T. VEGF protects brain against focal ischemia without increasing blood-brain permeability when administered intracerebroventricularly. J Cereb Blood Flow Metab. 2005;25(9): 1111-8. https://doi.org/10.1038/sj.jcbfm.9600109.

27. Schabbauer G, Tencati M, Pedersen B, Pawlinski R, Mackman N. PI3K-Akt pathway suppresses coagulation and inflammation in endotoxemic mice. Arterioscler Thromb Vasc Biol. 2004;24(10): 1963-9. https://doi.org/10.1161/01.ATV.0000143096.15099.ce.

28. Morrison A, Yan X, Tong C, Li J. Acute rosiglitazone treatment is cardioprotective against ischemia-reperfusion injury by modulating AMPK, Akt, and JNK signaling in nondiabetic mice. Am J Physiol Heart Circ Physiol. 2011;301(3):H895-902. https://doi.org/10. 1152/ajpheart.00137.2011.

29. Biscetti F, Straface G, Arena V, Stigliano E, Pecorini G, Rizzo P, et al. Pioglitazone enhances collateral blood flow in ischemic hindlimb of diabetic mice through an Akt-dependent VEGF-mediated mechanism, regardless of PPAR gamma stimulation. Cardiovasc Diabetol. 2009;8:49. https://doi.org/10.1186/1475-2840-8-49.

30. Kernan WN, Viscoli CM, Furie KL, Young LH, Inzucchi SE, Gorman M, et al. Pioglitazone after ischemic stroke or transient ischemic attack. N Engl J Med. 2016;374(14):1321-31. https:// doi.org/10.1056/NEJMoa1506930. 